\title{
Spagna e Italia in dialogo nell'Europa del Cinquecento
}

\author{
Marzia Faietti
}

\begin{abstract}
Viaggiare insegna lo spaesamento, a sentirsi sempre stranieri nella vita, anche a casa propria, ma essere stranieri fra stranieri è forse l'unico modo di essere veramente fratelli. Per questo la meta del viaggio sono gli uomini.
\end{abstract}

Claudio Magris, L'infinito viaggiare, Milano 2005

Secondo appuntamento con il disegno in Spagna dopo l'esposizione aperta nel 2016 alla Real Academia de Bellas Artes de San Fernando a Madrid, ${ }^{1}$ la mostra Spagna e Italia in dialogo nell'Europa del Cinquecento ${ }^{2}$ è stata concepita in modo assai diverso rispetto alla venue madrilena. Due, soprattutto, le differenze sostanziali: il taglio cronologico circoscritto al secolo XVI rispetto al periodo più ampio documentato a Madrid compreso tra la metà circa del Cinquecento e la metà del Settecento; la presenza di opere di varia tipologia (dipinti, sculture, medaglie, incisioni e libri antichi) dovute ad artisti anche italiani, assenti invece alla Real Academia de Bellas Artes de San Fernando dove erano in mostra solo disegni di autori spagnoli selezionati dal fondo grafico degli Uffizi ${ }^{3}$ - questi ultimi, viceversa, a Firenze sono stati integrati da qualche foglio di altre collezioni, italiane e spagnole-. Nelle intenzioni dei tre curatori della mostra, Corinna T. Gallori, Tommaso Mozzati e chi scrive, le varianti apportate consentivano di focalizzare meglio un'area geografico-culturale di strategica importanza in un periodo assai significativo della storia europea e di illustrare la circolazione culturale dell'epoca con la selezione di opere sia di artisti italiani attivi in Spagna (come Pellegrino Tibaldi), sia di importanti interlocutori di un dialogo a distanza (quali Sebastiano del Piombo, Daniele da Volterra e Giorgio Vasari).

\footnotetext{
1. Navarrete Prieto, B.; Alonso Moral, R., (a cura di), I segni nel tempo. Dibujos españoles de los Uffizi, catalogo della mostra, 12 maggio-24 luglio 2016, Real Academia de Bellas Artes de San Fernando, Madrid. Madrid: Fundación Mapfre, 2016.

2. FAIETti, M.; Gallori, C.T.; MozzATI, T., (a cura di), Spagna e Italia in dialogo nell'Europa del Cinquecento, catalogo della mostra. Firenze: Giunti, 2018. Il presente articolo riprende sinteticamente i contenuti principali del saggio da me pubblicato all'interno di quel catalogo; FAIETTI, M., «Per una storia globale del disegno. "El dibujo en España" nell'età di Carlo V e Filippo II», ibidem, pp. 18-37.

3. La più cospicua raccolta di fogli spagnoli presenti oggi agli Uffizi è pervenuta al museo fiorentino nel 1866 grazie alla donazione di Emilio Santarelli; si veda da ultimo ALONso MORAL, R., «El escultor Emilio Santarelli, coleccionista de dibujos», in Navarrete Prieto, B.; Alonso Moral, R., (a cura di), I segni nel tempo..., pp. 62-77, con bibliografia precedente.
} 
1. Jusepe (José) de Ribera, detto lo Spagnoletto Noli me tangere, penna e

inchiostro su

carta,

$23,5 \times 17,8 \mathrm{~cm}$.

Gabinetto dei

Disegni e delle

Stampe, Gallerie degli Uffizi,

Firenze.

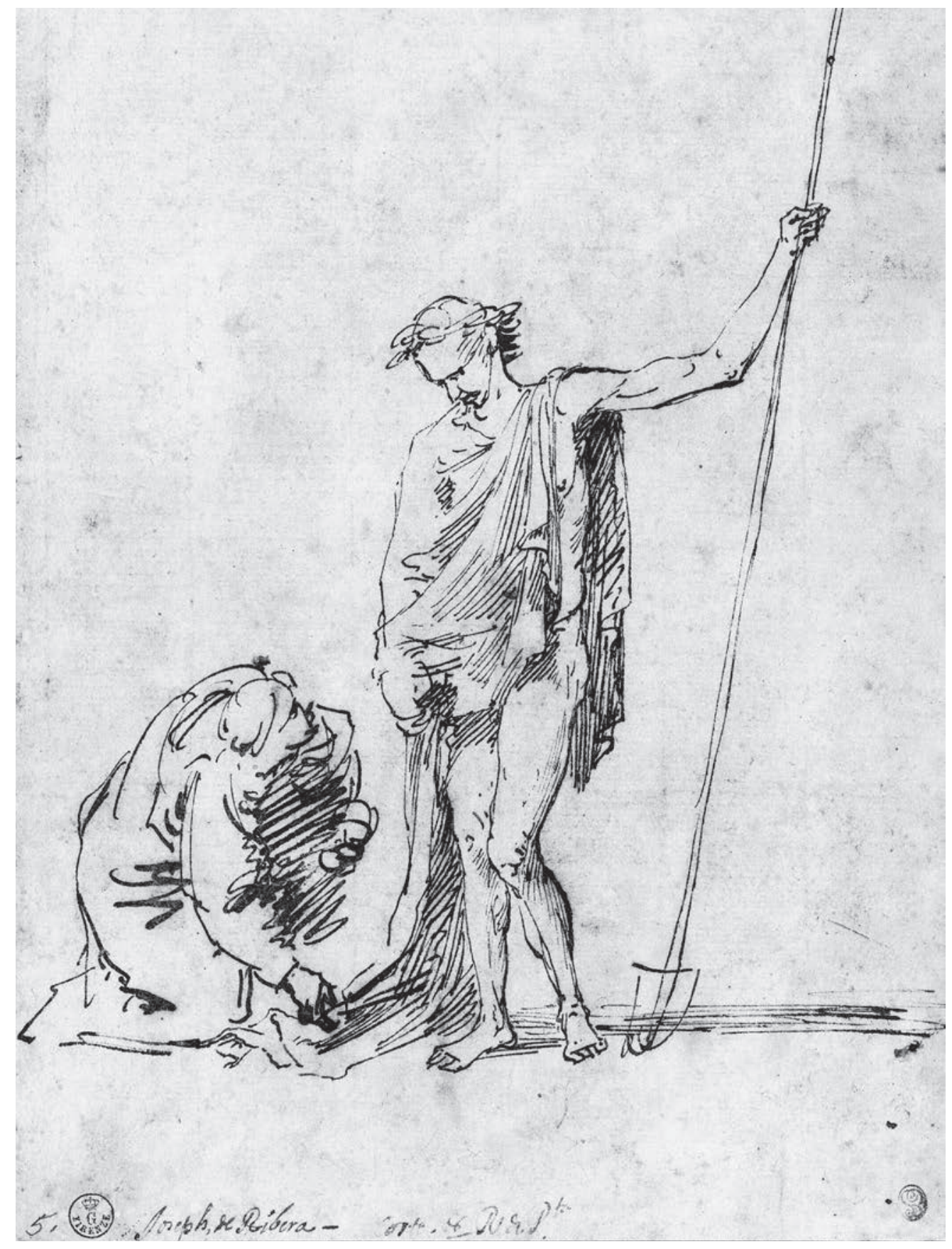

Le ragioni che mi hanno indotto a modificare l'esposizione agli Uffizi sono tuttavia anche altre, legate a questioni di natura essenzialmente metodologica come indicavo già in chiusura del mio intervento nel volume madrileno ${ }^{4}$ accennando al «caso» piuttosto emblematico di Jusepe (José) de Ribera, detto lo Spagnoletto (illustrazioni 1 e 2), artista nato nel 1591 e dunque assente a Firenze per motivi cronologici. Unico pittore spagnolo a essere rappresentato nella collezione fiorentina sin dalle sue origini, lo Spagnoletto venne classificato in ordine alfabetico tra gli autori

4. Faietti, M., «La “Scuola Spagnuola” de Pasquale Nerino Ferri (1895-1901)», in Navarrete Prieto, B.; Alonso MoRAL, R., (a cura di), I segni nel tempo..., pp. 78-87 ( p. 86). 


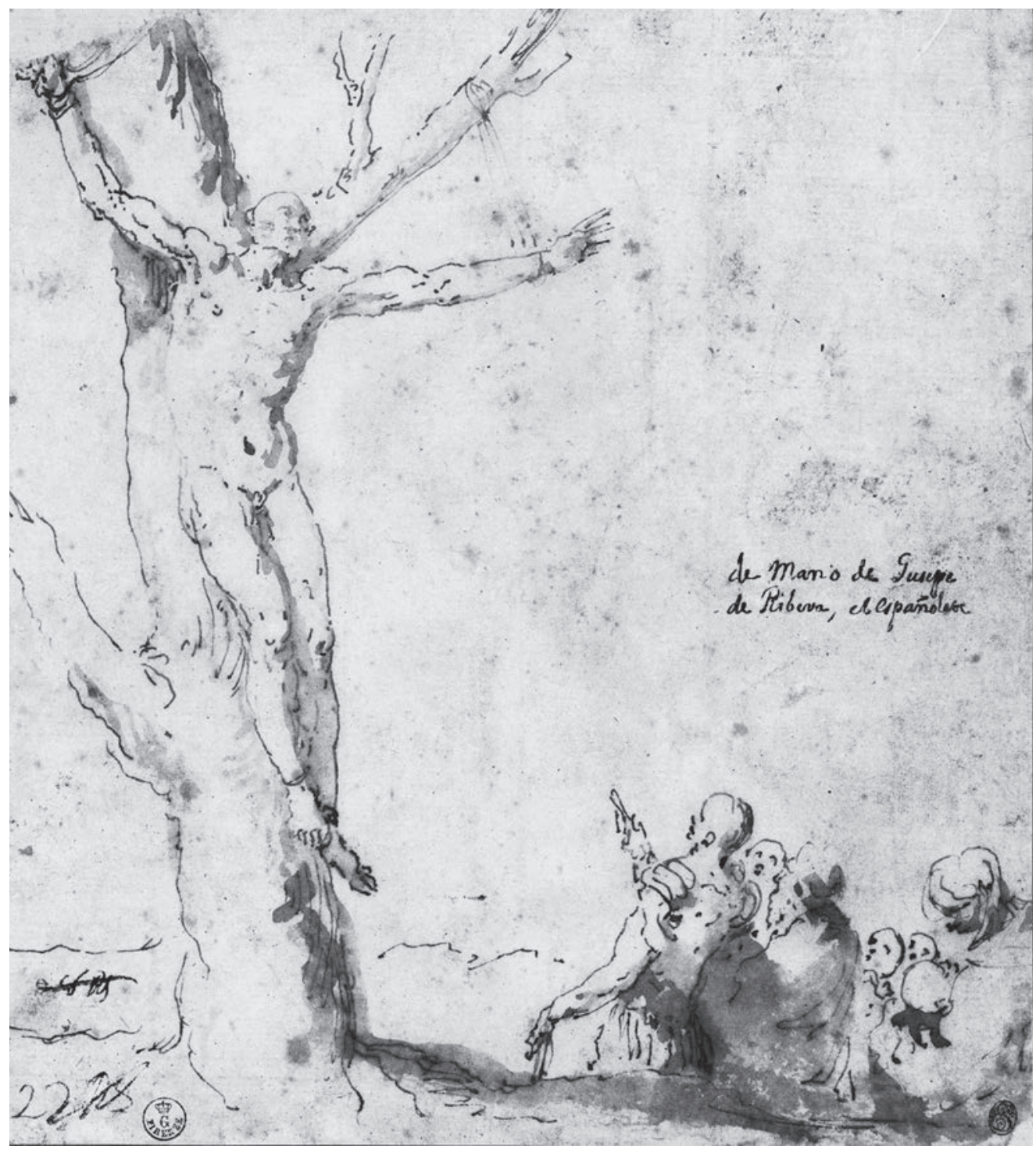

2. Jusepe (José) de Ribera, detto lo Spagnoletto Crocifissione di Policrate, penna e inchiostro, pennello e inchiostro diluito su carta, $22,5 \times 20,3 \mathrm{~cm}$. Gabinetto dei Disegni e delle Stampe, Gallerie degli Uffizi, Firenze.

italiani da Filippo Baldinucci (il primo curatore delle raccolte medicee per conto del cardinal Leopoldo de' Medici) ${ }^{5}$ nella sua Listra de' Nomi de' Pittori, di mano de' quali si hanno Disegni, stampata l'8 settembre 1673 , ma aggiornata sino al 1 agosto 1675 con note manoscritte autografe apposte su una copia oggi alla Biblioteca Nazionale Centrale di Firenze. ${ }^{6}$ Probabilmente, a favorire la sua assimilazione agli artisti italiani (e in particolare partenopei) fu il fatto che fogli dello Spagnoletto agli Uffizi provengono anticamente da Napoli. Poco meno di due secoli dopo, Luigi Scotti, pittore aggregato alla direzione della Reale Galleria di Firenze e autore di un catalogo dei disegni datato 30 ottobre 1832, distribuì i fogli assegnati all'artista in due cartelle rispettivamente

5. Sul cardinal Leopoldo, collezionista di disegni, si veda da ultimi Aliventi, R.; Rin Bettina, L. DA; FaIETti, M.; Grasso, M.; SASSI, R., «Una “muta historia”: la storia dell'arte per immagini nella collezione di disegni di Leopoldo de’ Medici», in Conticelli, V.; Gennaioli, R.; Sframeli, M. (a cura di), Leopoldo de' Medici. Principe dei collezionisti, catalogo della mostra, 7 novembre 2017-25 febbraio 2018, Palazzo Pitti, Tesoro dei Granduchi, Firenze. Livorno: Sillabe, 2017, pp. 116-131.

6. Baldinucci, F., Listra de' Nomi de' Pittori, di mano de' quali si hanno Disegni, Biblioteca Nazionale Centrale di Firenze, postillato 97, 1673-1675; si veda Fileti MAZzA, M., Storia di una collezione. Dai libri di disegni e stampe di Leopoldo de' Medici all'età moderna. Firenze: Leo S. Olschki, 2009, p. 234. I disegni erano quattro nel 1673, cui se ne aggiunsero altri nove entro il 1675 . 
afferenti alle scuole spagnola e napoletana. ${ }^{7}$ In assenza di descrizioni e numeri di inventario, non possiamo però conoscere i criteri di quella differente distribuzione, sui quali sarebbe invece stato interessante soffermarsi. Solo verso la fine dell'Ottocento, in ogni caso a partire dal 1890, con Pasquale Nerino Ferri, direttore dell'allora Gabinetto Disegni e Stampe della Reale Galleria degli Uffizi, lo Spagnoletto venne considerato un autore esclusivamente spagnolo. ${ }^{8}$

Ribera, dunque, può essere assunto a caso paradigmatico delle incoerenze e contraddizioni in cui ci imbattiamo nella catalogazione delle opere quando, passando dalla dimensione critica dello studio monografico su un autore all'esposizione analitica della sua produzione in strumenti miscellanei come, per esempio, i cataloghi di mostre, privilegiamo criteri di descrizione tassonomica che frammentano il fenomeno culturale in singoli segmenti, significativi ma non esaustivi. ${ }^{9} \mathrm{Ci}$ può allora capitare di impiegare in modo scarsamente flessibile alcuni concetti basilari acquisiti dalla storiografia artistica, ${ }^{10}$ restringendo in maniera eccessiva il campo in cui collocare o interpretare la multiforme attività di un autore. Viceversa, si può involontariamente causare qualche incertezza o dubbio ai nostri lettori cui capita di imbattersi, mentre sfogliano cataloghi di mostre o di collezioni permanenti, in talune figure che rappresentano alternativamente scuole diverse e magari con le stesse opere.

L'esistenza di artisti con esperienze multiformi e maturate in ambienti geografico- culturali diversi - a volte affini, a volte decisamente disomogenei tra loro- sollecita alcune questioni inerenti al concetto storiografico di scuola (la cui piena consapevolezza venne raggiunta solo tra la prima e la seconda decade del Seicento) e induce a valutarne l'efficacia come criterio di ordinamento museografico. Si è giustamente osservato che «strumenti di classificazione stilistica di orizzonte diverso, ma di origine comune» come la scuola intesa nella sua accezione genealogico-banausico (vale a dire, il gruppo di allievi e collaboratori di bottega che assorbirono in modi diversi la lezione del maestro) e la scuola in senso locale (ad esempio, la scuola spagnola) «derivano [entrambi] dall'esigenza collezionistica, oltre che critico-storiografica, di distinguere stili diversi, raggruppandoli però in famiglie omogenee, che rispettino griglie dotate di coordinate spazio-temporali»."

La rivoluzione informatica ha consentito la reperibilità simultanea di molteplici opere e differenti artisti e favorito rapidi incroci di dati e informazioni relativi a contesti culturali assai distanti tra loro; nel far ciò ha stimolato la verifica dei presupposti conoscitivi del sistema classificatorio per scuole e della sua efficacia sul piano dell'organizzazione museografica dei materiali. Mi riferisco in particolare alla scuola nazionale, poiché il processo di rivisitazione della nozione di stato nazionale in atto nell'ambito della storiografia ${ }^{12}$ ha indubbie ricadute anche sul piano della storia dell'arte, particolarmente evidenti in un'area geografico-politica caratterizzata da una complessa ricchezza di scambi e da incroci di esperienze culturali come l'Europa.

7. Scotтi, L., Catalogo dei Disegni originali dei Pittori, Scultori, et Architetti, che si conservano nella celebre Collezione esistente nella Imperiale e Reale Galleria di Firenze, Gallerie degli Uffizi, Gabinetto dei Disegni e delle Stampe, Firenze, ms. datato 30 ottobre 1832 (con aggiunte sino al 1837), c. 40, cartelle 10 e 156.

8. FERRI, P.N., Catalogo riassuntivo della raccolta di disegni antichi e moderni posseduta dalla R. Galleria degli Uffizi di Firenze. Roma: Principali Librai, 1890, pp. 862-863.

9. Si veda FAIETTI, M., «I disegni di scuola napoletana agli Uffizi dall'Ottocento a Walter Vitzthum», in SoLINAs, F.; Schütze, S. (a cura di), Le Dessin Napolitain, atti del colloquio internazionale, 6-8 marzo 2008, École Normale Supérieure, Parigi. Roma: De Luca, 2010, pp. 297-312 (pp. 308-309)

10. Sul tema si vedano i diversi contributi presenti in DaCosta Kaufmann, T.; Pilliod, E. (a cura di), Time and place. The geohistory of art. Aldershot: Ashgate 2005, nonché DACosta KaUfMann, T., «Introduction», pp. 1-19, per il rapporto tra arte e geografia, con bibliografia ragionata.

11. Perini, G., Arte in Europa, 1550-1650. L'età dei conflitti religiosi. Torino: UTET, 2008, pp. 219-220 (citazione a p. 219); vedi anche Bologna, F., La coscienza storica dell'arte d'Italia. Introduzione alla «Storia dell'arte in Italia». Torino: UTET, 1982, pp. 123-163.

12. Si veda in particolare SPINELli, A., La crisi degli stati nazionali. Bologna: Il Mulino, 1991; HoBsBAwn, E.J., Il secolo breve. Milano: Rizzoli, 1995; GidDEns, A., Il mondo che cambia. Come la globalizzazione ridisegna la nostra vita. Bologna: Il Mulino, 2000; HobsBawn, E.J., La fine dello Stato. Milano: Rizzoli, 2007. 
Mi sembra, dunque, emergere l'opportunità di concepire cataloghi aperti, volti a rettificare sia l'enfasi municipalistica — o regionale - dello schema narrativo delle Vite, sia l'ordinamento troppo rigidamente incentrato sulla scuola nazionale, entrambi conseguenze di determinati momenti storici piuttosto che criteri dotati di validità universale. Le opere di un artista sono il frutto finale di un'ininterrotta circolazione di idee e di forme. Scrivere sul disegno in Spagna, come su quello praticato in Italia o in qualsiasi altro luogo, significa ampliare costantemente gli orizzonti di ricerca, giungendo a sfiorare il punto di rottura della nozione di scuola nella sua accezione genealogico-banausico e locale, senza tuttavia rinunciare al recupero filologico del tessuto artistico di un luogo specifico e all'analisi della trasmissione del sapere nelle diverse botteghe. Solo dall'insieme coordinato di due movimenti dialetticamente opposti (la ricerca improntata inizialmente secondo i sistemi identificativi tradizionali delle scuole e la progressiva elaborazione di criteri metodologici che indirettamente giungano ad ampliare quei sistemi) nasce, a mio avviso, la piena comprensione di fenomeni storico-artistici articolati e sedimentati.

Luigi Scotti pareva aver accolto la nozione della scuola in senso locale nel suo catalogo datato 1832, come del resto anche Pasquale Nerino Ferri, che peraltro dovette attingere le sue informazioni basilari da compilazioni biografiche settecentesche, ad esempio El museo pictórico di Antonio Palomino per gli spagnoli ${ }^{13}$ e le Vite de' pittori, scultori ed architetti napoletani di Bernardo De Dominici per i napoletani (queste ultime riedite tra il 1840 e il 1846, in tempi non lontani dai suoi). ${ }^{14}$ Quando Ferri mise a punto nel 1893 un nuovo allestimento dei disegni in Galleria, intese seguire una sistemazione geografico-cronologica per scuole, di cui diede puntualmente conto in opere manoscritte e a stampa. ${ }^{15}$ Quella suddivisione era senza dubbio favorita dal contesto storico: nell'epoca dell'affermazione degli stati nazionali, le scuole europee affiancavano la scuola italiana rafforzando in modo indiretto la rappresentatività di quella giovane nazione ancora in parte da costruire, ma già in procinto di entrare nel primo conflitto mondiale.

Soltanto alla scuola italiana, comunque, Ferri riservò la distinzione al suo interno in diverse scuole regionali, mentre considerò le scuole straniere esclusivamente su base nazionale, compresa quella spagnola dove, viceversa, la suddivisione in aree culturali regionali rimane un criterio di identificazione ancora oggi in uso. Viene da chiedersi se la distinzione in diverse scuole italiane (a loro volta sottocategorie della scuola italiana all'interno della quale venivano tutte ricomprese) fosse determinata dal numero complessivo dei disegni presenti in collezione e dall'indiscussa ricchezza del patrimonio culturale del nostro paese o se, al contrario, fosse la conseguenza di una mancata conoscenza delle particolari accezioni locali del disegno riscontrabili anche nelle altre scuole. In effetti, solo con la generazione successiva a Ferri si fece strada una maggiore consapevolezza del disegno praticato in Spagna. Il curatore che gli successe, Odoardo Hillyer Giglioli, aveva tra l'altro conosciuto Diego Angulo Íñiguez in occasione della sua visita agli Uffizi, rimanendo in seguito in corrispondenza con lo studioso spagnolo. Ai suoi tempi fortunatamente non esisteva più l'allestimento dei disegni in Galleria, dal momento che nel 1909 era stata inaugurata una sala adibita alle esposizioni temporanee del Gabinetto dei Disegni e delle Stampe, in seguito all'incisivo riordinamento delle collezioni promosso da Corrado Ricci. La creazione di quello spazio espositivo, determinata dalla raggiunta consapevolezza del danno arrecato dall'azione prolungata della luce sulle opere cartacee, pose radicalmente fine agli allestimenti permanenti che si erano succeduti dal 1854 in poi. Non sappiamo se la

13. Per l'ipotesi di altre pubblicazioni ancora a disposizione di Ferri si veda FaIETTI, M., «Per una storia globale...», nota 17, p. 35 .

14. FAIETTI, M., «I disegni di scuola napolitana...», p. 307.

15. FerRi, P.N., Catalogo riassuntivo..., pp. 68-70; idem, «I disegni della Regia Galleria degli Uffizi», Arte e Storia, XII, 9, 1893, pp. 65-70; idem, Catalogo dei disegni, cartoni e bozzetti esposti al pubblico nella Reale Galleria degli Uffizi ed in altri musei di Firenze, Gallerie degli Uffizi, Gabinetto dei Disegni e delle Stampe, Firenze, 1895-1901. 
decisione museografica di rinunciare alle esposizioni permanenti fosse all'origine di un diverso criterio di riorganizzazione dei materiali e degli artisti, con il conseguente annullamento, sugli scaffali dei depositi, della separazione tra autori italiani e stranieri -come accade oggimotivata fino a poco più di un decennio prima dall'adozione del principio espositivo per scuole. Sappiamo però che la registrazione dei disegni per ordine alfabetico degli artisti aveva radici lontane, rifacendosi al primo documento ufficiale della collezione, la Listra di Baldinucci; qui, peraltro, gli autori «Oltramontani» erano distinti dagli italiani e collocati in fondo a ogni lettera alfabetica, salvo poche eccezioni.

Circa una ventina d'anni dopo Firenze conobbe un rinnovato interesse verso i disegni spagnoli e maturò una più adeguata comprensione della complessità dell'argomento. In seguito al suo viaggio nella città toscana, tra il 1927 e il 1928 Angulo Íñiguez pubblicava nella rivista Archivo Español de Arte y Arqueología tre studi relativi alla collezione degli Uffizi, uno dei quali incentrato su un foglio di Vicente Carducho, gli altri due dedicati alla raccolta più in generale. ${ }^{16}$ Lo studioso spagnolo non mancò di manifestare la sua particolare gratitudine al curatore fiorentino Giglioli. Quest'ultimo, rispetto a Ferri, poteva disporre di un maggior numero di pubblicazioni uscite nel frattempo, come si evince da diversi suoi appunti manoscritti. ${ }^{17}$ Tra queste, ricorrono frequentemente alcuni contributi fondamentali di August L. Mayer licenziati nel corso degli anni dieci, dove erano citati anche fogli degli Uffizi: l'articolo Der Racionero Alonso Cano und die Kunst von Granada (1910) e le due opere intitolate rispettivamente Geschichte der spanischen Malerei (1913) e Dibujos originales de maestros españoles. 150 apuntes y estudios de artistas del siglo XVI hasta el siglo XIX (1920).$^{18}$ Tuttavia, il rapporto senza dubbio più intenso che si arguisce dalla lettura delle note manoscritte è proprio quello stabilito con Angulo Íñiguez, di cui, tra l'altro, Giglioli possedeva pagine di appunti spediti dall'Università di Granada, risalenti al dicembre del 1924. Non è dato sapere se egli destinasse le sue annotazioni (non prive di osservazioni personali) a una pubblicazione poi non uscita, oppure se vi facesse ricorso soltanto per comprendere meglio quel fondo di disegni presente in collezione, tanto ricco di autori madrileni del XVII secolo da calamitare l'attenzione di uno studioso assai attratto dal Seicento e dal Settecento italiani, come lui. ${ }^{19}$

Nelle note manoscritte, Giglioli usa frequentemente l'espressione scuola spagnola allo stesso modo del suo predecessore Ferri e come, in epoca ancora precedente, appariva sia nei documenti relativi all'acquisto di disegni dai fratelli Felice, Giovanni e Luigi Michelozzi (1779) ${ }^{20}$ sia nel catalogo di Scotti. Prima di allora non abbiamo traccia di tale locuzione anche in conseguenza del fatto che artisti provenienti dalla Spagna non erano contemplati nei più antichi documenti finora reperiti, il che non significa che non vi fossero presenti sotto mentite spoglie. ${ }^{21}$

16. Angulo IÑIgueZ, D., «Un dibujo de Vicente Carducho en el Museo de los Uffizi, de Florencia», Archivo Español de Arte y Arqueología, 3, 1927, pp. 95-96; idem, «Dibujos españoles en el Museo de los Uffizi», Archivo Español de Arte y Arqueología, 3, 1927, pp. 341-347; idem, «Dibujos españoles en el Museo de los Uffizi, II», Archivo Español de Arte y Arqueología, 4, 1928, pp. 45-55.

17. Gallerie degli Uffizi, Gabinetto dei Disegni e delle Stampe, Firenze, ms. Elenco consistenza, n. 67.

18. Giglioli cita, ad esempio, Mayer, A.L., «Der Racionero Cano und die Kunst von Granada», Jahrbuch der Preussisches Kunstsammlungen, XXX, 1910, pp. 1-29; idem, Geschichte der Spanischen Malerei, 2 voll. Leipzig: Klinkhardt \& Biermann, 1913; idem, Dibujos originales de maestros españoles. 150 apuntes y estudios de artistas del siglo XVI hasta el siglo XIX, 2 voll. Leipzig - New York: The Hispanic Society of America, 1920 (edizione in lingua spagnola della precedente edizione in lingua tedesca licenziata a Lipsia nel 1915 e non citata dal curatore fiorentino).

19. Su questo aspetto si legga FAIETTI, M., «Dalla Firenze di Lanzi alla Bologna di Malaguzzi Valeri. Vicissitudini del disegno bolognese del Settecento", in Frommel, S. (a cura di), Crocevia e capitale della migrazione artistica: forestieri a Bologna e bolognesi nel mondo (secolo XVIII), atti del convegno internazionale di studi, 22-24 maggio 2012, Bologna. Bologna: Bononia University Press, 2013, pp. 377-396.

20. Su tale acquisto si veda da ultimo, con bibliografia, NAVArRete PrIETo, B., «El triunfo del dibujo español en la Galería de los Uffizi», in Navarrete Prieto, B.; Alonso Moral, R., (a cura di), I segni nel tempo..., pp. 12-43 (pp. 14-17).

21. Per qualche esempio si legga Faietti, M., «La “Scuola Spagnuola”...», p. 85. 
Nel 1930 Sánchez Cantón ebbe il merito di inserire nei volumi dei suoi Dibujos Españoles ${ }^{22}$ le fotografie raccolte da Angulo Íñiguez durante il viaggio fiorentino e non tutte illustrate nei suoi articoli. Ma, commenta severamente Alfonso Pérez Sánchez nell'Introduzione al catalogo dell'esposizione fiorentina del 1972, Sánchez Cantón finì per scegliere «senza dubbio per scrupolo di principiante, soltanto quei disegni che, o anonimi o erroneamente attribuiti, egli era riuscito a identificare, senza invece accogliere né [sic] riprodurre quelli che erano già correttamente catalogati». ${ }^{23}$ Viceversa, la mostra a Firenze del 1972 si poneva agli esordi di una rinnovata stagione di ricerche sul disegno spagnolo che coinvolgeva diversi paesi oltre alla Spagna: una fioritura di studi che in seguito non si sarebbe mai esaurita fino a proseguire, con rinnovato vigore, sino ai nostri giorni. Tralascio qui una disamina dei titoli principali per la quale rinvio a quanto ho scritto nel catalogo della mostra fiorentina, ${ }^{24}$ limitandomi a osservare che a monte di quei fondamentali strumenti di lavoro si colloca una pubblicazione di taglio veloce e divulgativo data alle stampe nel 1970, all'interno della collana I disegni dei maestri curata da Walter Vizthum per i Fratelli Fabbri Editori. Si trattava di un saggio intitolato Gli spagnoli da El Greco $a$ Goya, in realtà assai significativo fin dalle considerazioni iniziali:

I disegni degli artisti spagnoli sono stati e sono, in un certo qual modo, dei grandi sconosciuti. Scarsamente studiati e raramente riprodotti, sono stati oggetto di una sorta di sistematica dimenticanza e disdegno, e si è arrivati quasi a dimenticarne l'esistenza e il valore. ${ }^{25}$

Nella traduzione italiana di Lucia Cerutti la parola «disdegno» (non conosco purtroppo il testo originale in spagnolo) risulta piuttosto forte e la dice lunga sullo sviluppo degli studi in quegli anni e sulla rivendicazione di un ruolo del disegno spagnolo nel contesto della storia della grafica occidentale, una rivendicazione che, tra l'altro, si prefiggeva di smentire il pregiudizio della mancanza di interesse nei confronti dell'esercizio del disegnare da parte degli artisti spagnoli, considerati pittori «alla prima». ${ }^{26}$ Non mi sembra privo di interesse il fatto che un'analoga intitolazione fosse stata scelta pochi anni addietro per l'esposizione Spanische Zeichnungen von El Greco bis Goya: ${ }^{27}$ aperta nel 1966 ad Amburgo e corredata da un succinto catalogo curato da Wolf Stubbe, quella mostra aveva avuto il merito di accostare tra loro fogli conservati presso la notevole collezione della Hamburgher Kunsthalle ${ }^{28}$ ad altri pervenuti da tre importanti istituzioni madrilene, il Museo Nacional del Prado, la Biblioteca Nacional de España e la Real Academia de Bellas Artes de San Fernando. Il nome El Greco introduce sin dall'inizio un principio di contraddizione: artista di origine greca, peraltro mai dimenticata come testimonia il soprannome, vissuto anche in Italia prima di approdare a Toledo, seppe fondere, nelle sue suggestive opere, tradizioni artistiche dei luoghi della propria esperienza biografica e professionale, giungendo a elaborare un'arte personale e originalissima, una specie di sintesi antico-moderna. Un artista, dunque, che parve assimilare l'essenza stessa dei paesi dove aveva trascorso la sua vita e maturato le sue conoscenze, esprimendola in un linguaggio artistico non precisamente localizzabile in un determinato luogo; e, nondimeno, un artista che

22. SÁnchez Cantón, FJ., Dibujos españoles, 5 voll. Madrid: Hauser y Menet, 1930.

23. Pérez Sánchez, A.E., «Introduzione», in Pérez SÁnchez, A.E., Mostra di disegni spagnoli, catalogo della mostra, 1972-1973, Gabinetto Disegni e Stampe degli Uffizi, Firenze. Firenze: Leo S. Olschki, 1972, p. 5.

24. FAIETTI, M., «Per una storia globale ...», pp. 24-26.

25. PÉrez SÁnchez, A.E., Gli spagnoli da El Greco a Goya. Milano: Fratelli Fabbri, 1970; fa parte della collezione pubblicata a Vizthum, W. (a cura di), I disegni dei maestri; citazione a p. 5.

26. Si veda anche PÉrez Sánchez, A.E., «Introduzione», in PÉrez Sánchez, A.E., Mostra di disegni..., p. 7.

27. StubBe,W. (a cura di), Spanische Zeichnungen von El Greco bis Goya, catalogo della mostra, ottobre-novembre 1966, Hamburger Kunsthalle, Amburgo. Amburgo: Hans Christians, 1966.

28. Si veda a questo proposito il recente catalogo di HoffMann-SAMLAND, J. (a cura di), Dibujos Españoles en la Kunsthalle de Hamburgo, catalogo della mostra, 30 ottobre 2014 - 8 febbraio 2015, Museo Nacional del Prado, Madrid. Dallas - Madrid - Amburgo: Museo Nacional del Prado, 2014. 
3. Domenikos Theotokopoulos, detto El Greco Veduta di Toledo, c. 1599-160o, olio su tela,

$121,3 \times 108,6 \mathrm{~cm}$. The Metropolitan Museum of Art, New York.

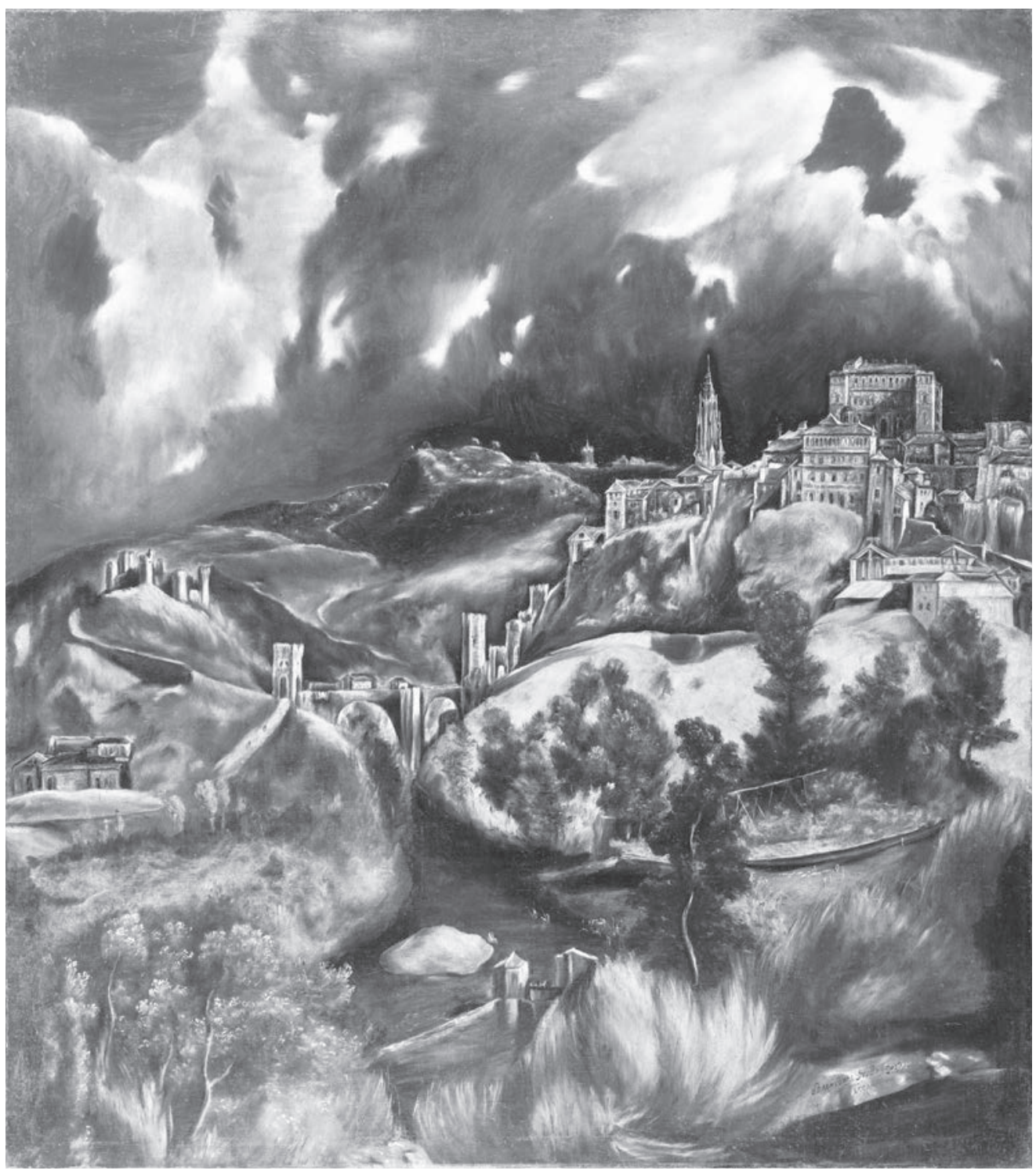

viene collocato più spesso tra gli autori spagnoli. Persino lo storico Fernand Braudel, nel suo La Méditerranée et le Monde Méditerranéen à l'époque de Philippe II, parlando del «Mediterraneo aereo» che sovrasta il «Mediterraneo di terra e d'acqua», ${ }^{29}$ gli dedicò un breve e intenso passaggio che lo legava indissolubilmente a Toledo: «Sopra Toledo, grazie all'umidità atlantica, l'inverno è responsabile di quei cieli offuscati, patetici, tempesta e luce, dipinti dal Greco» (illustrazione 3 )..$^{30}$

Artisti come Ribera e Il Greco rafforzano le mie convinzioni: il Museo ideale dovrebbe essere oggi impegnato, sotto il profilo museografico, nel superamento delle interpretazioni più rigidamente cristallizzate del termine scuola, lasciando alle spalle la sua accezione nazionale e, soprattutto, abbattendo le artificiose dogane culturali del nazionalismo ottocentesco. L'obiettivo finale che mi sembra necessario perseguire è l'apertura del museo a una dimensione più

29. Braudel, F., Civiltà e imperi del Mediterraneo nell'età di Filippo, 2 voll. Torino: Einaudi 2010 (1949), p. 238, traduzione italiana di C. Pischedda, aggiornata alla quinta edizione francese del 1982.

30. Ibidem, p. 240 
ampia e a una circolazione globale della cultura che siano coerenti con gli scambi ininterrotti fra paesi europei ed extra-europei favoriti dalle vicissitudini storiche, economiche e sociali. Il progetto scientifico sotteso a questa mostra va, dunque, ben oltre l'intento classificatorio del fondo grafico degli Uffizi e l'individuazione di una fisionomia del disegno spagnolo o, ancora meglio, del disegno praticato in Spagna (Historia del dibujo en España è l'incipit significativo del titolo di un'opera fondamentale di Pérez Sánchez del 1986), ${ }^{31}$ nei diversi aspetti assunti, senza ombra di dubbio, nelle varie aree geo-culturali di quel paese. La classificazione delle singole opere in riferimento alle differenti regioni artistiche e l'identificazione di alcune caratteristiche ricorrenti, e possibilmente precipue e identificative del disegno in Spagna, costituiscono dunque il primo traguardo del nostro percorso conoscitivo. Nelle tappe successive, precedute da un «lavoro sul campo» riguardante i nuovi materiali grafici, tale percorso tenderà al riconoscimento degli aspetti culturali più generali che differenziano e accomunano tra loro i paesi afferenti all'area mediterranea. Si intende che in questo caso si sta parlando di una porzione piuttosto limitata di quell'area: non certo del più Grande Mediterraneo suggestivamente rievocato da Braudel e dallo studioso misurato sui parametri della storia, le cui dimensioni oltrepassano di gran lunga i confini tracciati dai geografi, dai geologi e dai biogeografi, ${ }^{32}$ ma una zona circoscritta del Mare Interno. Il che non significa che il nostro sguardo sui fenomeni artistici maturati in quelle regioni e in quel periodo debba essere necessariamente circoscritto, dal momento che quei fenomeni si inscrivono all'interno di vicende nodali della storia europea. La mostra si ripromette, dunque, di contestualizzare il modo di disegnare di autori spagnoli o attivi in Spagna nell'età di Carlo V e di Filippo II, $^{33}$ facendo riferimento a un tratto minore del Mare Interno, che potremmo definire il Piccolo Mediterraneo, dal quale sono separate e unite tra loro Italia e Spagna.

Il soggetto presenta tutte le caratteristiche di un case study dal punto di vista metodologico per i flussi culturali che in Spagna si succedettero incessantemente dal secolo precedente, manifestandosi dapprima in forma centripeta e poi centrifuga, e interessando progressivamente il Nord e il Sud dell'Europa, l'Africa, l'Asia e l'America centro-meridionale. A metà del quinto decennio del Novecento, qualche anno prima della pubblicazione di Braudel (peraltro già fissata nelle sue linee generali, se non scritta per intero, sin dal 1939), ${ }^{34}$ Angulo Íñiguez si era spinto sulle rotte oceaniche con la sua Historia del arte hispanoamericano. ${ }^{35}$

Rientrando nelle acque più pacifiche del Piccolo Mediterraneo, occorre fare ancora qualche considerazione relativamente al disegno in Spagna, cui altre seguiranno man mano che conosceremo sempre meglio i materiali grafici elaborati in aree più vaste.$^{36}$ In primo

31. PÉrez SÁnchez, A.E., Historia del dibujo en España. De la Edad Media a Goya. Madrid: Cátedra, 1986; sul significato del titolo si intrattiene anche Mena MARQuÉs, M.B., La efímera historia del dibujo español, in NAVARRETE PRIETO, B.; Alonso Moral, R., (a cura di), I segni nel tempo..., p. 45. Si veda anche, a tal proposito, CAVI, S. DE , «Benito Navarrete Prieto (dir.), "I segni nel tempo. Dibujos españoles de los Uffizi"», Goya, 361, 2017, pp. 352-354; l'autrice menziona inoltre il testo fondamentale di MARÍAs, F., El largo siglo XVI. Los usos artísticos del Renacimiento español. Madrid: Taurus, 1989, in relazione alla reinterpretazione dell'identità artistica e architettonica del Rinascimento in Spagna come fenomeno di lunga durata delle tradizioni artistiche locali.

32. Braudel, F., Civiltà e imperi..., vol. I, pp. 166-236 (pp. 166-169).

33. Sui rapporti tra Italia e Spagna durante il regno di Filippo II, si veda LotTI, L.; VILLARI, R. (a cura di), Filippo II e il Mediterraneo. Roma-Bari: Laterza, 2003.

34. Braudel, F., Civiltà e imperi..., vol. I, p. Xxx (prefazione alla seconda edizione francese del 1966).

35. Angulo IÑIguez, D., Historia del arte hispanoamericano. Barcellona - Buenos Aires: Salvat, 1945. Vorrei inoltre ricordare il volume di Kubler, G.; SorIA, M., Art and Architecture in Spain and Portugal and their american Dominions 1500 to 180o. Harmondsworth: Penguin Books, 1959, che considerava anche l'arte e l'architettura del Portogallo e delle sue colonie americane.

36. Per il disegno delle arti decorative in chiave trasversale tra Portogallo, Spagna, Italia, Malta e Grecia si veda CAvi, S. DE (a cura di), Dibujo y Ornamento: Trazas y dibujos de artes decorativas entre Portugal, España, Italia, Malta y Grecia. Estudios en Honor de Fuensanta García de la Torre, atti del convegno Dibujar las Artes Aplicadas: dibujo de ornamentación para platería, mayólica, mobiliario, arquitectura efímera y retablística entre Portugal, España e Italia (siglos XVI-XVIII), 5-8 giugno 2013, Universidad de Córdoba. Córdoba - Roma: Diputación de Córdoba - De Luca, 2015. Si veda anche l'ottima 
luogo sarebbe un esercizio inutile tracciare le caratteristiche di un disegno spagnolo autonomo, per esempio con lo scopo di limitare la portata del ruolo propulsivo esercitato nel suo sviluppo dagli artisti italiani. La prima condizione di un'effettiva autonomia è infatti la capacità di assorbire stimoli differenti, tanto più che nella ricostruzione delle dinamiche culturali non ci sono propriamente né vincitori (in questo caso, gli italiani con la supremazia nell'arte del disegno), né vinti (gli spagnoli con la subordinazione al disegno degli italiani). Nel nostro processo di abbattimento di vecchi pregiudizi, ci è d'aiuto qualche caso di filologia testuale: ogni studioso di arte italiana ricorda le parole spese da Giorgio Vasari nella Vita di Tiziano, uscita nell'edizione Giuntina del 1568, sull'incapacità dei veneziani nel disegnare, ${ }^{37}$ un'accusa priva di fondamento che, in fondo, risulta piuttosto simile a quella rivolta per lungo tempo agli artisti spagnoli. È sufficiente una lettura critica del testo vasariano e del progetto sotteso alle Vite per comprendere come quell'opinione contenesse il manifesto programmatico della necessità della formazione accademica e la pretesa dell'eccellenza didattica della neonata Accademia del Disegno fiorentina (1563), cui Vasari voleva assicurare il primato nelle fasi progettuali che precedono e indirizzano l'esecuzione pittorica.$^{38}$ In realtà, come ben sappiamo, esistono modi diversi di disegnare e nessuno di essi può vantare un primato in termini assoluti.

In quella porzione circoscritta del Mediterraneo costituita dalla Spagna e dall'Italia, gli artisti parteciparono a uno scambio ininterrotto e, a seconda dei momenti e delle esperienze personali, orientato verso direzioni molteplici, piuttosto che univoche. Non vorrei parlare di influssi, condividendo a tal riguardo le giuste riserve a suo tempo avanzate da Michael Baxandall, ${ }^{39}$ ma di una traiettoria di andata e ritorno, durante la quale i caratteri originali del punto di partenza subiscono sensibili metamorfosi e contaminazioni reciproche - ho in mente, ad esempio, «los italianos escurialenses» Federico Zuccaro (illustrazione 4), Luca Cambiaso (illustrazione 5), Pellegrino Tibaldi (illustrazioni 6, 7 e 8) e Giovanni Battista Castello, detto il Bergamasco-.${ }^{40}$ Ecco, allora, che studiare il disegno in Spagna nel Cinquecento ci induce a intraprendere un viaggio senza sosta, destinato ad allargare i confini geo-culturali e ad abbattere le barriere pregiudiziali.

A riprova della necessità di uno studio del disegno in Spagna in una prospettiva globale (prospettiva che peraltro si attaglia al disegno tout court per la sua intrinseca adattabilità alla circolazione su ampia scala), vorrei concludere con un esempio piuttosto significativo in cui mi sono imbattuta diversi anni fa. ${ }^{41}$ Riguarda un autore spagnolo attivo a Roma fra il 1516 e il 1518, nei cantieri di Raffaello nella Stufetta e nella Loggetta del cardinal Bibbiena nonché nelle Logge Vaticane, che a Bologna ricoprì un ruolo rilevante nella diffusione di un'idea compositiva con ogni probabilità derivata da idee raffaellesche. Pedro Machuca —è di lui che parlo- rivestì quella funzione tramite un foglio, oggi conservato al Louvre, con la Deposizione dalla croce (illustrazione 9), la cui cronologia ha oscillato tra il soggiorno italiano, il periodo iniziale del

recensione di BoubLI, L., «Dibujo y Ornamento: Trazas y dibujos de artes decorativas entre Portugal, España, Italia, Malta y Grecia. Estudios en Honor de Fuensanta García de la Torre. Sabina de Cavi», Revue de l’art, 197, 2017, pp. 82-83.

37. VASARI, G., Le Vite de' più eccellenti pittori, scultori e architettori nelle redazioni del 1550 e 1568 [BETTARINI, R.; BAROCCHI, P. (ed.)]. Firenze: Sansoni, 1987, edizione 1568, p. 156.

38. FAIETti, M., «Giorgio Vasari's Life of Titian: Critical Misinterpretations and Preconceptions Concerning Venetian Drawing», in Whistler, C. (a cura di), Drawing in Venice. Titian to Canaletto, catalogo della mostra, 15 ottobre 2015 - 10 gennaio 2016, Ashmolean Museum, Oxford. Oxford: Ashmolean Museum - University of Oxford, 2015, pp. 38-49.

39. Baxandall, M., Patterns of Intention. On the Historical Explanation of Pictures. New Haven - London: Yale University Press, 1985, pp. 58-62.

40. La definizione è di Pérez Sánchez, A.E., Museo del Prado. Catálogo de dibujos. Dibujos españoles siglos XV-XVII. Madrid: Alfiz 1972, p. 13.

41. Faietti, M., «Pedro (Machuca), Biagio (Pupini), Innocenzo (Francucci) e la "Deposizione di Cristo"», in Boschloo, A.W.A.; Grasman, E.; SMAn, G.J.van Der (a cura di), "Aux Quatre Vents". A Festschrift for Bert W. Meijer. Firenze: Centro Di, 2002, pp. 49-54. 


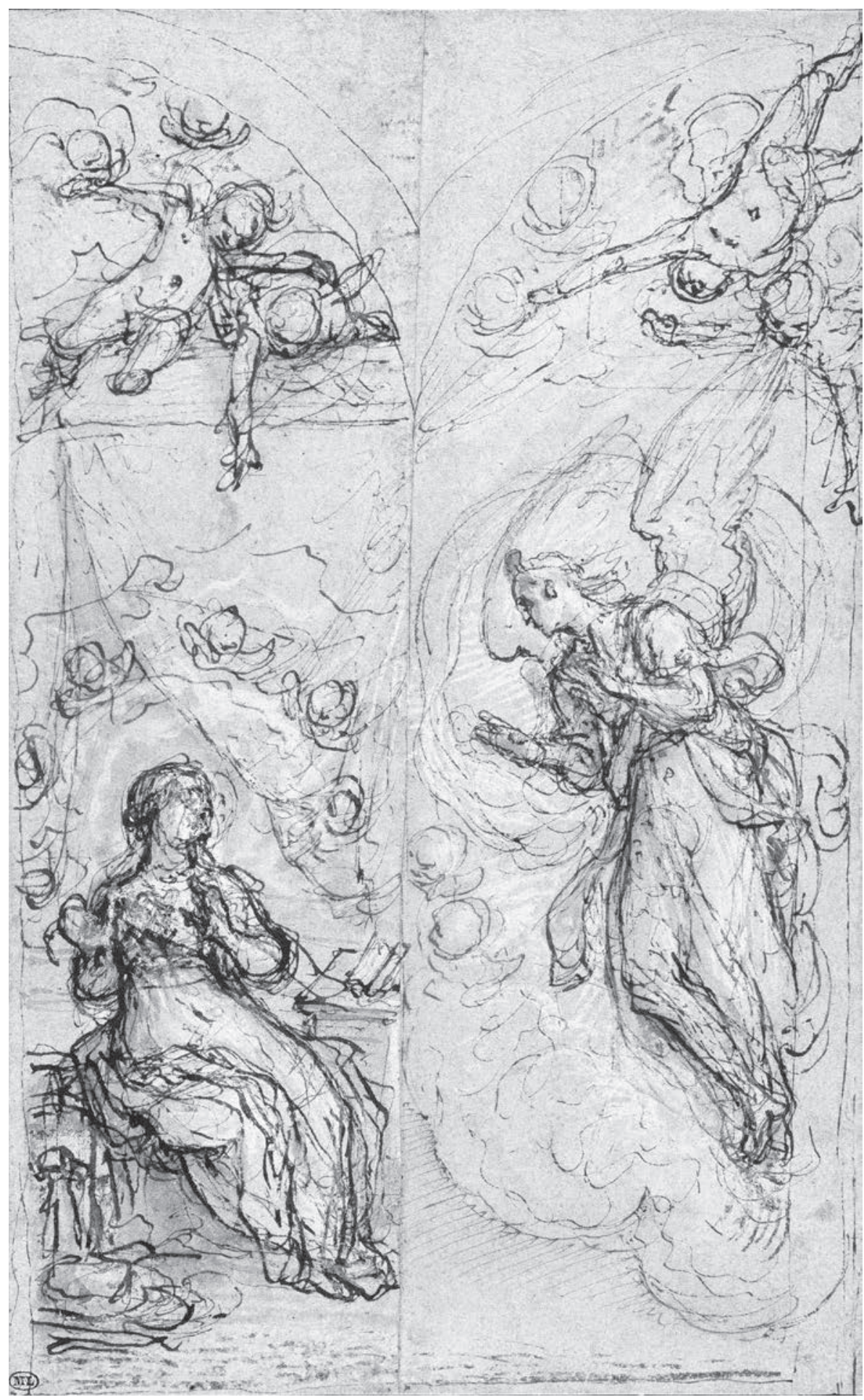

4. Federico

Zuccari

Studio per

l'Annunciazione

dell'altare

dell'Annunziata

nella basilica

dell'Escorial,

c. 1586, penna

e inchiostro,

inchiostro diluito

su carta blu,

$25 \times 15,4 \mathrm{~cm}$.

Département des

Arts graphiques,

Musée du

Louvre, Parigi.

rientro in Spagna — nel 1520 Machuca risulta stabilitosi definitivamente a Granada - o anche qualche tempo dopo. ${ }^{42}$ In quel disegno l'artista forse interpolava tra loro uno o più prototipi

42. Musée du Louvre, Paris, Département des Arts graphiques, inv. 6303; sul disegno si veda BouBLI, L., in BouBLI, L.; PÉRez SÁnchez, A.E. (a cura di), Dessins espagnols. Maîtres des XVI ${ }^{e}$ et XVII ${ }^{e}$ siècles, catalogo della mostra, 18 aprile-22 luglio 1991, Musée du Louvre, Parigi. Parigi: Réunion des Musées Nationaux, 1991, pp. 63-65, n. 13, con la precedente bibliografia; 
5. Luca

Cambiaso

Gloria dei beati,

1584, affresco,

volta del coro,

basilica,

monastero di

San Lorenzo de

El Escorial, San

Lorenzo de

El Escorial.

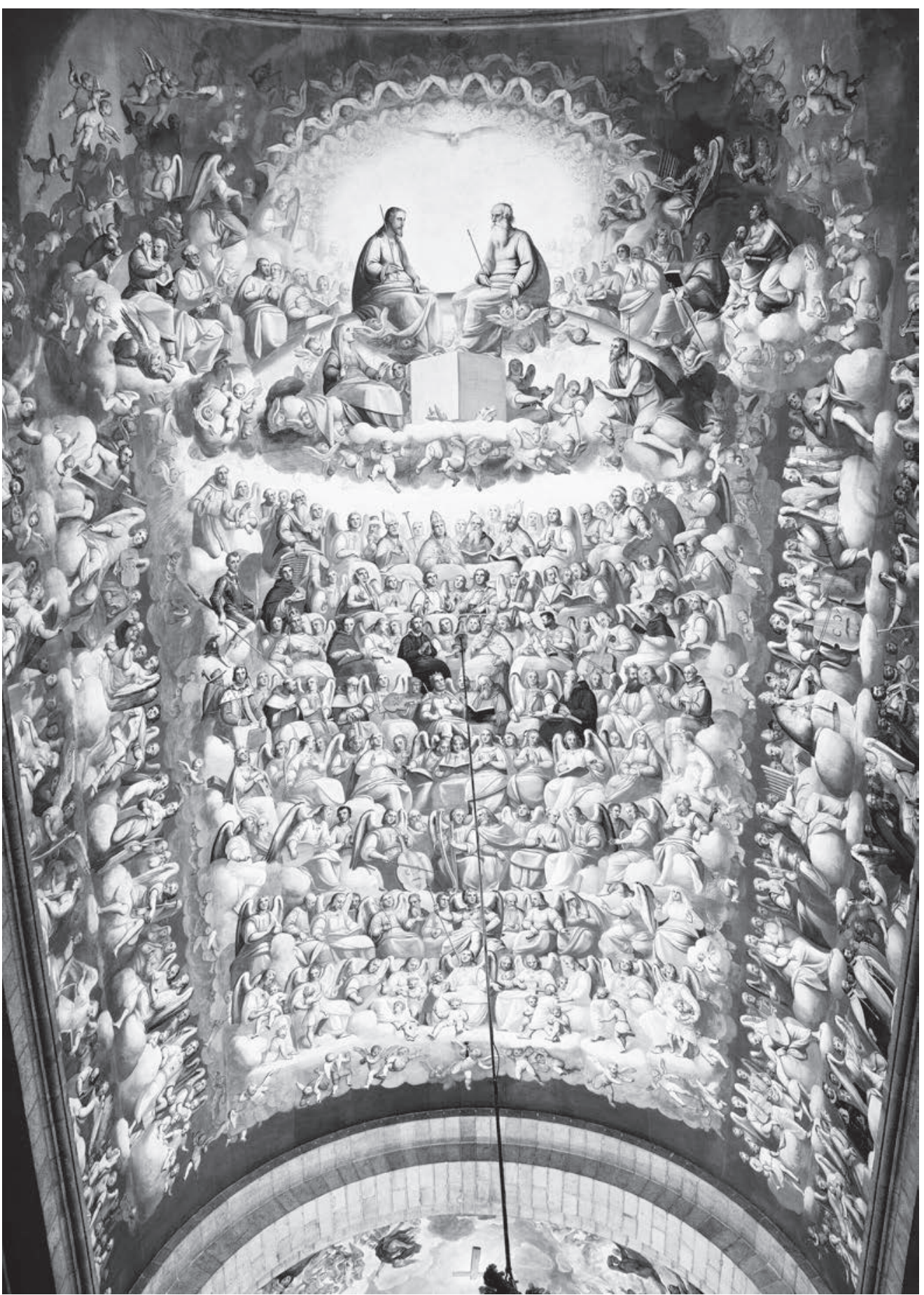

appresi nei cantieri romani di Raffaello, dando luogo a una composizione originale che ritroviamo nel suo dipinto con la Deposizione dalla croce ora al Prado. ${ }^{43}$ In realtà, quest'ultimo non

idem, Musée du Louvre. Département des Arts Graphiques. Inventaire Général des dessins. École Espagnole XVI ${ }^{e}$-XVIII ${ }^{e}$ siècle. Parigi: Réunion des Musées Nationaux, 2002, pp. 32-34, n. 12. Si veda anche la bibliografia indicata nella nota seguente.

43. Si leggano, con la bibliografia precedente, McDonALD, M.P., Renaissance to Goya: prints and drawings from Spain . Londra: The British Museum Press, 2012, p. 126; BouBLI, L., «Studio e invenzione: Pedro Machuca e Alonso Berruguete, disegnatori tra Italia e Spagna», in Mozzati, T.; NATALI, A. (a cura di), Norma e capriccio. Spagnoli in Italia agli esordi della 


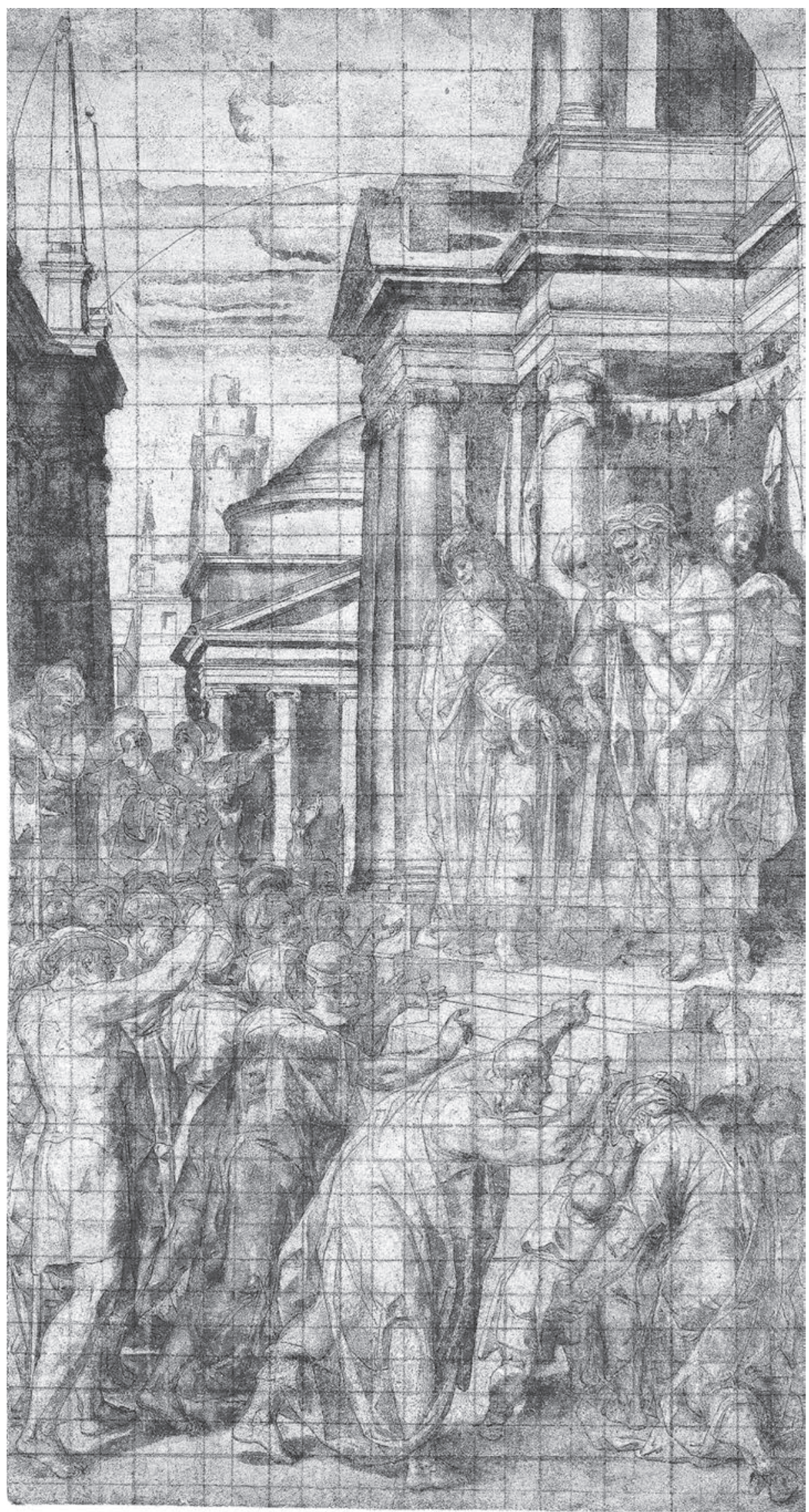

6. Pellegrino

Tibaldi

Cristo presentato

al popolo

per il chiostro

dell'El Escorial,

1587-1590,

penna,

inchiostro,

inchiostro diluito

su gesso nero,

arricchito

con bianco

$54,8 \times 29,3 \mathrm{~cm}$.

Department

of Prints and

Drawings, The

British Museum,

Londra. 
7. Pellegrino

Tibaldi

Cristo presentato

al popolo, c. 1586 ,

affresco, chiostro,

monastero di

San Lorenzo

de El Escorial,

San Lorenzo

de El Escorial

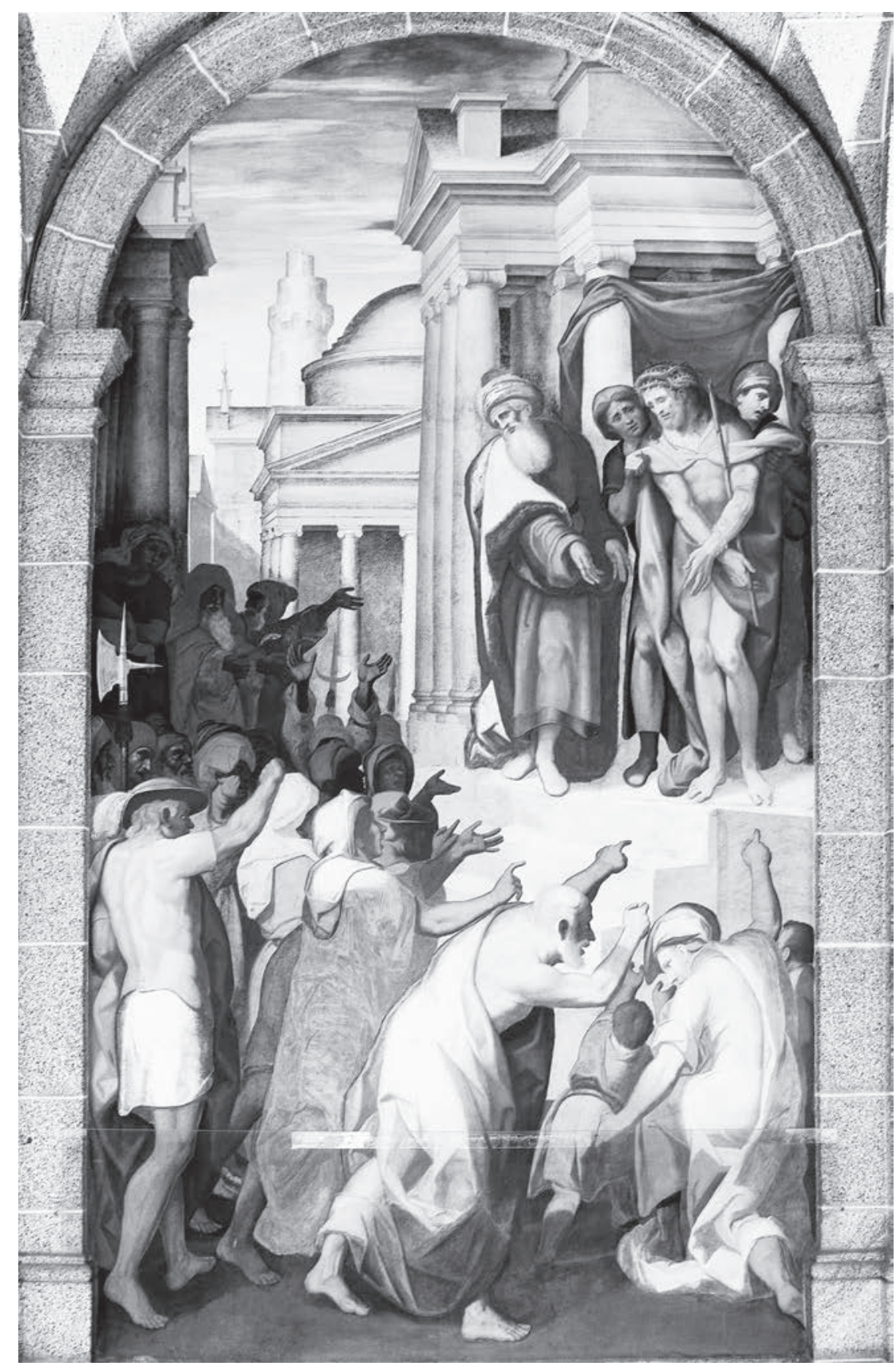

è la sola trasposizione pittorica del foglio parigino: ${ }^{44}$ esso, infatti, funse senza dubbio da modello per l'affresco omonimo riemerso a seguito di restauri nella sala dei Capitoli conventuali invernali, sita accanto al Refettorio nell'ex convento bolognese dei canonici regolari del Santissimo Salvatore (illustrazione 10). L’affresco mi sembra riferibile a Innocenzo Francucci, più noto

«maniera moderna», catalogo della mostra, 5 marzo - 26 maggio 2013, Galleria degli Uffizi, Firenze. Firenze: Giunti, 2013, pp. 146-155 (pp. 147-150); BisCEgLIA, A., «Roma e gli spagnoli agli inizi del Cinquecento: la Sistina, Raffaello, le Logge vaticane», in ibidem, pp. 106-119 (p. 117); Boubli, L., Le dessin en Espagne à la Renaissance. Pour une interprétation de la trace. Turnhout: Brepols, 2015, pp. 175-185; BIsCEgLIA, A., «Berruguete e Machuca: composizioni religiose», in FAIETTI, M.; GALLORI, C.T., Mozzati, T., (a cura di), Spagna e Italia in dialogo..., pp. 110-118 (pp. 115-116).

44. Per una versione presente in una collezione privata di Madrid e dovuta allo stesso Machuca si veda BouBLI, L., Le dessin en Espagne..., pp. 183 e note 95 e 185. 

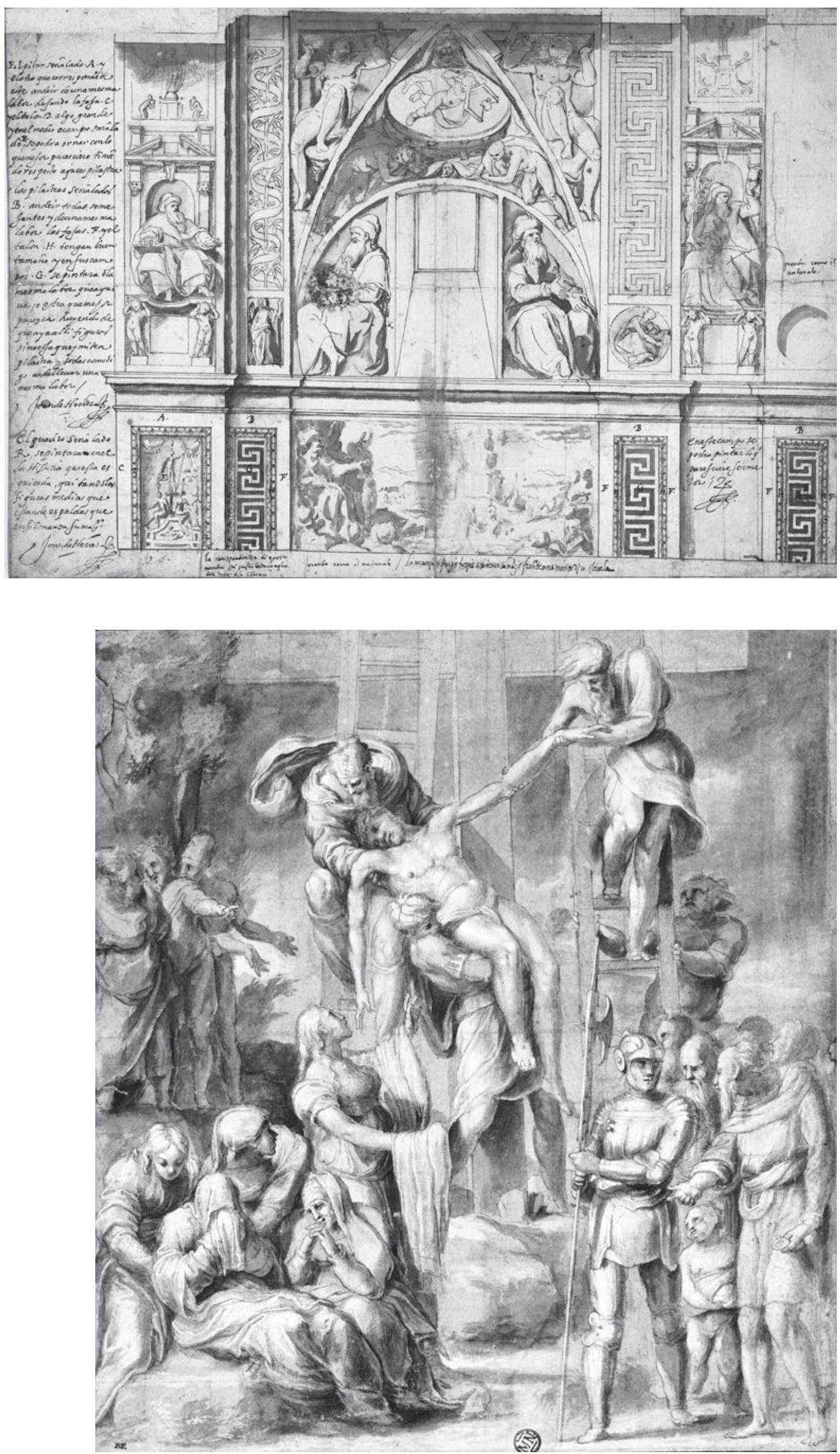

8. Pellegrino

Tibaldi

Studio per la decorazione della Biblioteca dell'El Escorial, 15881592, penna, inchiostro e inchiostro diluito su gesso nero, $33,2 \times 48,5 \mathrm{~cm}$. Department of Prints and Drawings, The British Museum, Londra.

9. Pedro

Machuca Deposizione dalla croce, c. 1517-1520, penna, inchiostro, acquerello grigio, lumeggiato a tempera bianca, matita nera, $31,5 \times 28,4 \mathrm{~cm}$. Département des Arts graphiques, Musée du Louvre, Parigi. 


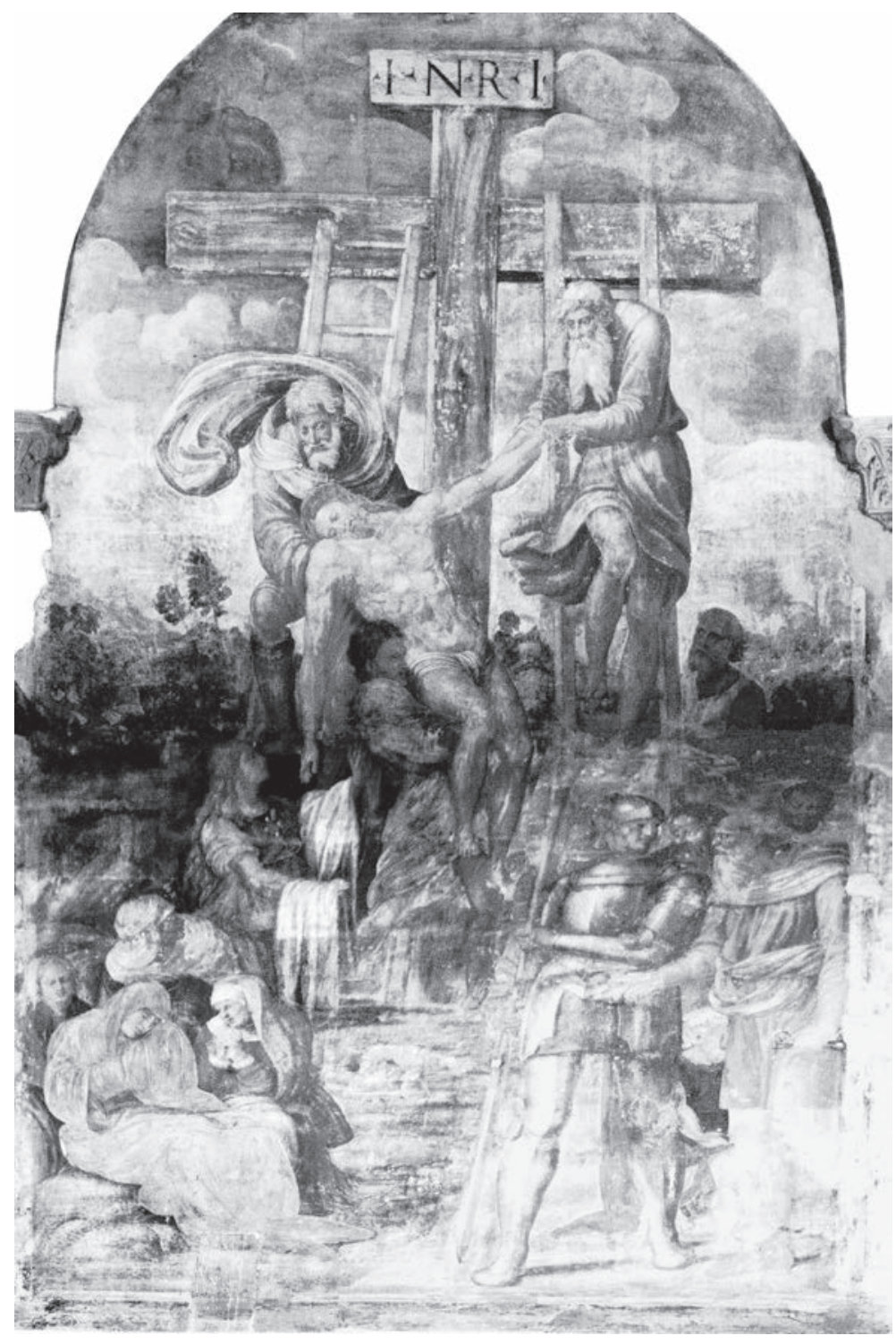

come Innocenzo da Imola, ${ }^{45}$ che l'avrebbe realizzato non molto dopo l'inizio dei lavori in San Salvatore (avviati nel 1517 nel plesso Refettorio-Libreria sul lato sud-orientale dell'attuale secondo cortile) e la sua attività nel convento degli olivetani di San Michele in Bosco, per i quali nel dicembre del 1517 aveva sottoscritto il contratto relativo agli affreschi della «sagrestia nuova» e alla Madonna con il Bambino in gloria e i santi Michele Arcangelo, Pietro e Benedetto destinata all'altar maggiore della chiesa. ${ }^{46}$ Un'esecuzione della $D e-$ posizione dalla croce successiva al 1517 e compresa nei primi anni venti mi pare probabile in relazione al linguaggio pittorico maturato in quegli anni da Innocenzo e alla circolazione del modello di Machuca tra altri artisti attivi nel convento, come Biagio Pupini. ${ }^{47}$ La parte inferiore della composizione ricompare, infatti, in un foglio di Pupini segnalato nel 1994 nella collezione Morton B. Harris ${ }^{48}$ mentre un ulteriore disegno presso l'Art Gallery of South Australia di Adelaide, attribuito di nuovo al pittore bolognese, richiama quell'invenzione ma con notevoli varianti e limitatamente alle figure di Cristo, Nicodemo e san Giuseppe d'Arimatea..$^{49}$ Non si può escludere che Pupini possa aver copiato integralmente e con fedeltà il modello di Machuca
10. Innocenzo Francucci, detto Innocenzo da Imola

Deposizione dalla croce, c. 1539, affresco, Sala del Capitolo d'inverno, ex convento di San Salvatore, Bologna. in un foglio finora sconosciuto e che i due disegni superstiti costituiscano una rivisitazione parziale e successiva del soggetto. In questo caso egli stesso potrebbe aver trasmesso la Deposizione di Pedro al collega Innocenzo. Le sue esperienze artistiche non contraddicono questa ipotesi: inizialmente vicino al linguaggio grafico di Polidoro da Caravaggio (artista messo a sua volta in relazione con Machuca) e con ogni probabilità più volte presente a Roma, con la sua copiosa attività disegnativa egli fu a Bologna il principale tramite delle idee elaborate nell'Urbe

45. Faietti, M., Pedro (Machuca)..., pp. 49-54.

46. Ora presso la Pinacoteca Nazionale di Bologna; si legga la scheda di Pedrini, C., in Bentini, J., CAMmarota, G.P.; Mazza, A.; Scaglietti Kelescian, D.; Stanzani, A. (a cura di), Pinacoteca Nazionale di Bologna. Catalogo Generale. 2. Da Raffaello ai Carracci. Venezia: Marsilio, 2006, pp. 69-71, n. 48.

47. Propende per una datazione più tarda, verso il 1539, BoubLI, L., «Studio e invenzione...», p. 148; idem, Le dessin en Espagne..., p. 176. Qui la datazione è proposta in modo ipotetico.

48. Sul disegno si veda la scheda di Wolk-Simon, L., in Griswold, W.M.; Wolk-Simon, L., Sixteenth-century Italian Drawings in New York Collections, catalogo della mostra, 11 gennaio-27 marzo 1994, The Metropolitan Museum, New York. New York: The Metropolitan Museum of Art, 1994, pp. 4-5, n. 3.

49. Inv. D717. Non avendolo potuto esaminare dal vivo, non sono in grado di stabilirne con certezza l'autografia. 
da Raffaello e dalla sua cerchia..$^{50}$ Ma non si deve neppure sottovalutare il ruolo autonomo di Innocenzo da Imola nella promozione di una nuova stagione di confronto con l'Urbinate, che stimolò nella città felsinea, tra la fine degli anni dieci e il primo quinquennio circa degli anni venti, una produzione di dipinti piuttosto interessante, soprattutto come fenomeno culturale, e a sua volta conseguenza di quella intensa circolazione di idee di Raffaello già avviata nello scorcio del primo decennio con i bulini di Marcantonio Raimondi e proseguita in seguito con copie disegnate. ${ }^{51}$ Verso la metà del terzo decennio Innocenzo, Pupini, Bartolomeo Ramenghi detto il Bagnacavallo e Girolamo da Carpi licenziavano dipinti derivati o ispirati a prototipi dell'Urbinate e della sua scuola; ricordo qui soltanto la Sacra Famiglia con sant'Elisabetta, san Giovannino e il fondatore del Collegio, Egidio Albornoz, desunta dalla Sacra Famiglia di Francesco I, realizzata da Biagio nel 1524 per una committenza spagnola nel chiostro superiore del Collegio di Spagna. ${ }^{52}$

Dunque, Pedro Machuca, tramite il suo disegno noto a Innocenzo da Imola e a Biagio Pupini, contribuì a quella laboriosa fucina del riciclaggio di composizioni raffaellesche, in particolare di ambito romano, sviluppatasi a Bologna soprattutto dalla fine degli anni dieci alla metà circa degli anni venti, quando anche i prestigiosi committenti del Collegio di Spagna parvero apprezzarla. Con la sua Deposizione dalla croce divenne infatti il capostipite di una nuova genealogia di immagini che discendeva da uno o più modelli raffaelleschi riproposti ai colleghi italiani tramite la sua personale rielaborazione, a sua volta destinata a diventare normativa. ${ }^{53}$

I disegni - oggetti in sé spesso complessi e intriganti e allo stesso tempo strumenti privilegiati per la circolazione di spunti inventivi, figurativi e decorativi grazie alle dimensioni ridotte e alla leggerezza del supporto cartaceo- sono tanto riconducibili sul piano artistico e storico all'ambiente che li ha prodotti, quanto facilmente decontestualizzabili dal loro ambito originario per essere nuovamente contestualizzati ogni volta che i viaggi e le esplorazioni culturali dell'artista, nonché le esigenze diversificate della committenza lo richiedano. Partendo da tali premesse, la mostra fiorentina ha inteso concorrere alla costruzione di una prospettiva globale del disegno mediante il caso emblematico del dibujo en España nell'età, soprattutto, di Carlo V e Filippo II.

50. Si leggano le schede di Faietti, M., in Faietti, M. (a cura di), Il Cinquecento a Bologna. Disegni dal Louvre e dipinti a confronto (con la collaborazione di CoRDELliER, D.), catalogo della mostra, 18 maggio-18 agosto 2002, Pinacoteca Nazionale, Bologna. Milano: Electa, 2002, pp. 124-135 (pp. 102-103), note 25-29.

51. Sul riciclaggio di idee raffaellesche a Bologna in quel periodo si veda FAIETTI, M., "Parmigianino "alter Raphael” ", in FAIETti, M. (a cura di), Raffaello, Parmigianino, Barocci: metafore dello sguardo, catalogo della mostra, 2 ottobre 2015-10 gennaio 2016, Musei Capitolini - Palazzo Caffarelli, Roma. Roma: Palombi, 2015, pp. 62-109 (pp. 68-69).

52. Musée du Louvre, Parigi, Département des Peintures, inv. 604; si veda MeYer zur CaPellen, J., Raphael. A Critical Catalogue of his Paintings. II: The Roman Religious Paintings, c. 1508-1520. Landshut: Arcos, 2005, pp. 29-31 e 170-177, nota 62, con la precedente bibliografia; HenRY, T.; JoAnnides, P., Raphaël. Les dernières années, catalogo della mostra, 12 giugno-16 settembre 2012, Museo Nacional del Prado, Madrid - Musée du Louvre, 11 ottobre 2012 - 14 gennaio 2013, Parigi. Malakoff: Éditions Hazan, 2012, pp. 135-143, nota 16 (Raphaël et atelier?).

53. Per la diffusione delle sue idee figurative e dei suoi motivi iconografici in ambito napoletano, presso Polidoro da Caravaggio, Marco Cardisco e altri ancora si veda MIERI, S. DE, «Pittura iberica nel vicereame (e ai suoi confini). Appunti su Pedro Machuca, "Domenico Spagnolo", Luis de Vargas e Luis de Morales», in GAETA, L. (a cura di), Napoli e la Spagna nel Cinquecento. Le opere, gli artisti, la storiografia. Galatina (Lecce): Mario Congedo, 2017, pp. 162-181, con ampia rassegna bibliografica sul soggiorno di Machuca a Napoli. 